\title{
HOT TOPICS Biologically plausible models of neural dynamics for rapid-acting antidepressant interventions
}

\author{
Jessica R. Gilbert ${ }^{1}$ and Carlos A. Zarate Jr. ${ }^{1}$ \\ Neuropsychopharmacology (2021) 46:231-232; https://doi.org/10.1038/s41386-020-00804-7
}

Recent advances in computational modeling have led to translational efforts to bridge neuroscience and psychiatry by applying novel computational methods to characterize abnormalities in brain systems that underlie psychiatric diseases, including depression. Such work could potentially help identify, categorize, and predict dimensional processes in depression [1], as well as understand how rapid-acting antidepressants modulate brain disease-state networks [2]. Progress in this field has led to recent attempts to use computational methods to characterize ketamine's antidepressant effects in depression [3, 4]. Ketamine is a noncompetitive $\mathrm{N}$-methyl-D-aspartate receptor (NMDAR) antagonist that is thought to exert its antidepressant effects through glutamatergic throughput via a-amino-3-hydroxy-5methyl-4-isoxazolepropionic acid receptors (AMPARs) [5]. Studies using dynamic causal modeling (DCM) - an approach that fits a biologically plausible model of neural dynamics to measured electrophysiological signals-have measured delayed (within $9 \mathrm{~h}$ of drug administration) changes in estimates of receptormediated and regional drive following antidepressant-level ketamine doses. Sets of coupled differential equations govern DCM dynamics, and model inversion-the fitting of parameterized mean-field neuronal models to data features-results in parameter estimates that govern unobservable neuronal states such as the decay times of specific receptor types and receptormediated connectivity between cell populations.

Recent work from our laboratory used a somatosensory stimulation task in tandem with magnetoencephalography to measure parameter changes in NMDAR and AMPAR drive between primary somatosensory cortex and frontal cortex in unmedicated individuals with treatment-resistant depression $6-9 \mathrm{~h}$ post ketamine and post saline placebo. During somatosensory stimulation, amplified top-down NMDAR drive was observed between frontal cortex and primary somatosensory cortex post ketamine [3]. This unexpected result has been attributed to upregulation and drug sensitization effects. Critically, in terms of antidepressant response, reduced NMDAR and AMPAR drive were both associated with better antidepressant response immediately, though only changes in AMPAR drive were associated with longer-term changes in antidepressant response 2 weeks post ketamine [3]. This second finding is in keeping with animal studies demonstrating long-term adaptation involving AMPAR upregulation [5].

Additional work assessing delayed effects (three to four hours post-ketamine) used electroencephalography and a visual grating task to measure changes in long-term potentiation.
Ketamine broadly amplified bottom-up drive between middle occipital gyrus and both inferior temporal cortex and superior parietal cortex, while asymmetrically attenuating and amplifying top-down drive between these same regions [4]. The biologically plausible model selected for this analysis did not include parameters modeling receptor-mediated effects, nor did it directly measure associations between modeled parameter estimates and antidepressant response.

Taken together, the evidence suggests that recent advances in modeling offer promise for uncovering how ketamine alters receptor-mediated connectivity between cell populations and even decay times of specific receptor types. However, considerable research is still needed to further develop and validate biophysical models of network dynamics. For example, models which parameterize local changes in excitation-inhibition could enhance translational efforts linking preclinical and human studies by measuring in silico associations between pyramidal cell disinhibition and antidepressant response, a potential biomarker of antidepressant efficacy [6]. Such knowledge could ultimately help develop next-generation, rapid-acting antidepressants by providing a more complete, mechanistic understanding of ketamine's antidepressant effects.

\section{FUNDING AND DISCLOSURES}

Funding for this work was supported by the Intramural Research Program at the National Institute of Mental Health, National Institutes of Health (IRP-NIMH-NIH; ZIAMH002857), by a NARSAD Independent Investigator Award to CAZ, and by a Brain and Behavior Mood Disorders Research Award to CAZ. These organizations had no further role in study design; in the collection, analysis, or interpretation of data; in the writing of the report; or in the decision to submit the paper for publication.

CAZ is listed as a co-inventor on a patent for the use of ketamine in major depression and suicidal ideation; as a co-inventor on a patent for the use of $(2 R, 6 R)$-hydroxynorketamine, $(S)$-dehydronorketamine, and other stereoisomeric dehydro and hydroxylated metabolites of $(R, S)$-ketamine metabolites in the treatment of depression and neuropathic pain; and as a co-inventor on a patent application for the use of $(2 R, 6 R)$-hydroxynorketamine and $(2 S, 6 S)$ hydroxynorketamine in the treatment of depression, anxiety, anhedonia, suicidal ideation, and post-traumatic stress disorders. He has assigned his patent rights to the US government but will share a percentage of any royalties that may be received by the

\footnotetext{
${ }^{1}$ Experimental Therapeutics and Pathophysiology Branch, National Institute of Mental Health, National Institutes of Health, Bethesda, MD, USA

Correspondence: Jessica R. Gilbert (jessica.gilbert@nih.gov)
}

Published online: 15 August 2020 
232

government. JRG has no conflict of interest to disclose, financial or otherwise.

\section{ACKNOWLEDGEMENTS}

The authors thank the 7SE research unit and staff for their support. Ioline Henter (NIMH) provided invaluable editorial assistance.

\section{AUTHOR CONTRIBUTIONS}

Both authors contributed equally to the conceptualization of the article, literature search, writing, and revision of this manuscript. Both authors approved the final version of the paper.

\section{ADDITIONAL INFORMATION}

Publisher's note Springer Nature remains neutral with regard to jurisdictional claims in published maps and institutional affiliations.

\section{REFERENCES}

1. Huys QJM, Maia TV, Frank MJ. Computational psychiatry as a bridge from neuroscience to clinical applications. Nat Neurosci. 2016;19:404-13.

2. Gilbert JR, Zarate CA. Electrophysiological biomarkers of antidepressant response to ketamine in treatment-resistant depression: gamma power and long-term potentiation. Pharmacol Biochem Behav. 2020;189:172856.

3. Gilbert JR, Yarrington JS, Wills KE, Nugent AC, Zarate CA Jr. Glutamatergic signaling drives ketamine-mediated response in depression: evidence from dynamic causal modeling. Int J Neuropsychopharmacol. 2018;21:740-7.

4. Sumner RL, McMillan R, Spriggs MJ, Campbell D, Malpas G, Maxwell E, et al. Ketamine enhances visual sensory evoked potential long-term potentiation in patients with major depressive disorder. Biol Psychiatry Cogn Neurosci Neuroimaging. 2019;5: 45-55.

5. Zanos P, Moaddel R, Morris PJ, Georgiou P, Fischell J, Elmer Gl, et al. NMDAR inhibition-independent antidepressant actions of ketamine metabolites. Nature. 2016;533:481-6.

6. Widman AJ, McMahon LL. Disinhibition of CA1 pyramidal cells by low-dose ketamine and other antagonists with rapid antidepressant efficacy. Proc Natl Acad Sci USA. 2018;115:E3007-16. 